\title{
Extrapyramidal and cerebellar syndrome with encephalopathy associated with cimetidine
}

\author{
Clive E. HANDLER* \\ B.Sc., M.R.C.P.
}

\author{
CHRISTOPHER P. BESSE \\ M.B. B.S.
}

\author{
ADRIAN O. WILSON \\ M.A., M.R.C.P.
}

\begin{abstract}
Department of Neurology, Charing Cross Hospital, Fulham Palace Road, London W6 8RF
\end{abstract}
\begin{abstract}
Summary
A possible case of cimetidine induced extrapyramidal and cerebellar features is reported. Although confusion is a well recognized toxic effect of cimetidine, other neurotoxic features are less common, especially in patients without evidence of renal or hepatic disease. Cimetidine should be used with great care and possibly in a reduced dose in the elderly as neuropsychiatric side effects may occur.
\end{abstract}

\section{Introduction}

Cimetidine, a specific histamine-2 receptor antagonist, is widely used in the treatment of peptic ulcer, and side effects, such as diarrhoea, muscular pain, rash and dizziness are relatively uncommon. Confusion is rare but has been described in elderly patients, usually with underlying renal or liver impairment. We are not aware of any reported cases of an extrapyramidal and cerebellar syndrome with an acute encephalopathy due to cimetidine and we report a possible case, since it gave rise to diagnostic difficulty.

\section{Case history}

A 72-year-old man presented to the Casualty Department with an acute confusional state. He had a past history of partial gastrectomy, hypertension and right cerebral infarction. Subsequent transient cerebral ischaemic episodes were treated with enteric-coated soluble aspirin. He had led a fully active life for the two years before presentation in August 1981. Then, while on a walking holiday, he was admitted to another hospital with haematemesis secondary to a bleeding duodenal ulcer. He was transfused, given cimetidine $1 \mathrm{~g}$ in divided doses and

*Present address: Dept of Cardiology, Guy's Hospital, London SE1 9RT. made a good recovery. The day following discharge (i.e. 18 days after starting cimetidine) he became acutely confused and was brought to Charing Cross Hospital by his wife. On examination, the patient was unable to provide a history, did not respond to verbal commands, and had a mild residual left pyramidal weakness. Initial biochemistry, haematology and blood gases were normal. Chest radiography showed evidence of emphysema and an electrocardiogram was normal.

He remained disorientated in place and time but remained on cimetidine. One week after admission he became more restless and agitated and developed tremor, cogwheel rigidity and marked retropulsion. He also manifested mild cerebellar signs with nystagmus on lateral gaze and intention tremor with pastpointing on finger-nose testing, predominantly in the right arm. He was treated with benzhexol $2 \mathrm{mg}$ three times daily but became more confused and had visual hallucinations, seeing 'berries'. He could not now recognize his wife and developed oro-facial dyskinesia and marked twitching. He was treated with chlormethiazole and thiamine but did not improve. The benzhexol was withdrawn. The following tests were normal: biochemical screen, full blood count, serum thyroxine, serum and red cell folate and serum B12. His liver function tests were normal apart from a low serum albumin of $28 \mathrm{~g} /$ litre. Three sets of blood cultures and a mid-stream urine sample were negative. An electroencephalogram showed bilateral posterior 4-6 Hz theta and 1-3 Hz delta activities with a maximal disturbance in the right parietal region, presumably related to his previous right cerebral hemisphere infarction. Computerized axial tomography of the brain showed dilatation of the lateral ventricles, mild cortical atrophy, and an area of diminished density in the right cerebral hemisphere consistent with an old infarction. Because of the extrapyramidal signs he had a one week trial of 
Madopar '125' (levodopa and benserazide) three times daily but remained markedly confused. Thirtythree days after admission the cimetidine was stopped. His mental state rapidly improved and over the next three weeks the tremor resolved and the rigidity slowly improved. The Madopar was discontinued and the patient's mobility increased.

\section{Discussion}

This patient developed an acute confusional state, visual hallucinations, twitching, cerebellar and extrapyramidal signs about 3 weeks after starting cimetidine in recommended oral dosage. He had no renal or hepatic impairment. Because of his known cerebrovascular disease, multi-infarct dementia may have caused the acute confusional state and possibly the extrapyramidal signs. There was, however, no evidence of hypovolaemic shock or hypoxia associated with his haematemesis. The acute onset of the widespread progressive neurological features nearly 3 weeks after the haematemesis, together with their resolution imply a cimetidine associated encephalopathy as the more likely explanation of the patient's illness. His improvement was sustained on stopping the anti-Parkinson's medication. In view of the severity of the neurological disturbance which responded to withdrawal of the cimetidine, we thought it clinically inappropriate to rechallenge him with the drug. The initial haematemesis may have been related to the prolonged aspirin ingestion.

Earlier reported cases have incriminated cimetidine as causing mental confusion (Grimson, 1977; Wood, Isaacson and Hibbs, 1978), visual hallucinations (Agarwal, 1978), and brain stem dysfunction (Cumming and Foster, 1978), usually within a few days of starting treatment. In some of these cases, however, other medical conditions existed which may have been contributory. A recent paper (Sonnenblick, Rosin and Weissberg, 1982) reviewed the neurological and psychiatric side effects of cimetidine. Mental confusion was present in over half the reviewed cases but reversible extrapyramidal and $\underset{\Phi}{\frac{2}{2}}$
cerebellar features are not recorded.

Cimetidine has been found in the cerebro-spinalc. fluid of patients with presumed normal meninges as $\Rightarrow$ well as those in whom the blood-brain-barrier has $\stackrel{?}{+}$ become more permeable due to meningitis or renalo failure and it may therefore have a central action $\frac{\bar{O}}{5}$ (Edmonds et al., 1979). It has been suggested that? cimetidine blocks histamine- 2 receptors in the central $\mathbb{\Phi}$ nervous system and this is endorsed in animal ${ }_{\infty}^{\infty}$ experiments. Because of the extrapyramidal features displayed in our patient, cimetidine may also have.effects on acetylcholine and dopaminergic receptors $\vec{\omega}$ in the brain.

We conclude that cimetidine must be used witho great care in the elderly, especially those with known 3 . cerebrovascular disease and renal or hepatic impair- $-\infty$ ment. Neuropsychiatric side effects may occur, even ${ }_{\infty}$ in recommended doses, and so a reduced dose of the drug should be given to those patients who are at risk from these complications.

\section{Acknowledgment}

We should like to thank Dr F. Clifford Rose for his permission to report this case.

\section{References}

Agarwal, S.K. (1978) Cimetidine and visual hallucinations. Journal of the American Medical Association (letter), 240, 214.

CUMmING, W.J.K. \& FosTER, J.B. (1978) Cimetidine induced 유 brainstem dysfunction (letter). Lancet, i, 1096.

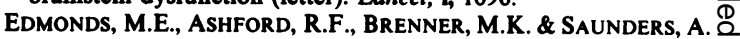

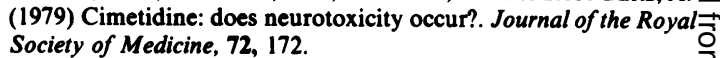

GrIMSON, T.A. (1977) Reaction to cimetidine (Letter). Lancet, i, 99.3

SONNENBliCK, M., Rosin, A.J. \& WeissberG, N. (1982) Neurological and psychiatric side effects of cimetidine. Report of three cases? with a review of the literature. Postgraduate Medical Journal, 58, 415.

WOOD, C.A., IsAaCsON, M.L. \& HibBS, M.S. (1978) Cimetidine and mental confusion. Journal of American Medical Association, $\frac{\sigma}{3}$ (letter). 239, 2550. 